\title{
Investment in Electricity Markets with Asymmetric Technologies
}

\author{
Talat S. Genc* \\ University of Guelph \\ Henry Thille \\ University of Guelph
}

July 13, 2009

\begin{abstract}
We study competition between hydro and thermal electricity generators under demand uncertainty. Producers compete in quantities and each is constrained: the thermal generator by capacity and the hydro generator by water availability. We analyze a two-period game emphasizing the incentives for capacity investments by the thermal generator. We characterize both Markov perfect and open-loop equilibria. In the Markov perfect equilibrium, investment is discontinuous in initial capacity and higher than it is in the open-loop equilibrium. However, since there are two distortions in the model, equilibrium investment can be either higher or lower than the efficient investment.
\end{abstract}

Keywords: Electricity markets; Dynamic game; Duopoly; Capacity investment.

JEL codes: D24, L13, L94

${ }^{*}$ Corresponding author. University of Guelph, Dept. of Economics, Guelph, ON, N1G2W1, Canada. Email: tgenc@uoguelph.ca. Phone: 1.519.824.4120 x56106. Fax: 1.519.763.8497 


\section{Introduction}

It is common to find alternative electricity generation technologies coexisting in a market. In many jurisdictions, electricity is generated from a mix of thermal (coal, oil, gas), nuclear, and hydro generation plants. Growth of the demand for electricity has meant that increased generation capacity is desired in many jurisdictions. Especially in deregulated markets, new generation capacity is not easily constructed for the larger, low marginal cost, generation technologies such as hydro and nuclear.

The main issue that we address in this paper is that of investment in new thermal generation capacity in the presence of a large hydro competitor. ${ }^{1}$ Following deregulation and in combination with demand growth, investment in new generation capacity occurred in many jurisdictions and continents (most significantly in Europe and South America). Given regulatory and environmental hurdles and substantial fixed costs, new hydro development is often not an option. In this case, new capacity is commonly provided by thermal plants. Since the incentive to invest in new capacity depends on the expected distribution of future prices, the extent to which incumbent hydro generation affects the distribution of prices will have an effect on investment in thermal capacity.

Although there has been much recent interest in models of electricity markets, there has not been much analysis of the implications for market performance when one of the producers has significant hydroelectric generation capability. Crampes and Moreaux [4] model an electricity market in which a hydro producer uses a fixed stock of water over two periods facing competition from a thermal producer. Bushnell [2] examines a Cournot oligopoly with fringe producers in which each producer controls both hydro and thermal generation facilities. Both hydro and thermal units face capacity constraints and the producers must decide how to allocate the available water over a number of periods. Scott and Read [17] develop a Cournot model of mixed hydro/thermal generation that is calibrated to the New Zealand wholesale electricity market. They focus on the effect of forward contracting, concluding that high levels of contracting are necessary to approach an efficient outcome. These papers all focus on the allocation of water by the hydro producers. In contrast, we focus on the longer term issue of investment by the thermal producer and abstract away from concerns about water use. ${ }^{2}$

In restructured electricity markets, the importance of having excess market capacity through capacity investments has been stressed (see Roques, Newbery, and Nuttall [16], Murphy and Smeers [13], Cramton and Stoft [5], Joskow [14], among others) for the sake of more competitive outcomes and, importantly, of system security so that possible supply disruptions offset and/or unexpected peak demand met. In the past, cost-of-service regulation enabled investors to recoup their investment costs through regulated rates. However, in deregulated markets investors are motivated by profits and it is the purpose of this paper

\footnotetext{
${ }^{1}$ We describe the low cost generation technology as hydroelectric throughout this paper. The model equally applies to any low marginal cost technology with a capacity constraint. As we are focusing on longer-term investment dynamics, we do not model shorter-term water flow dynamics explicitly.

${ }^{2}$ Papers which focus on competition between hydro generators include Ambec and Doucet [1], and Garcia, Reitzes and Stacchetti [6].
} 
to examine these incentives.

There are some recent papers that have examined investment incentives in electricity markets. Murphy and Smeers [13] examine generation capacity investments in open-loop and closed-loop Cournot duopolies. Each duopolist makes investments in production capacities. In the open-loop game, capacities are simultaneously built and sold in long-term contracts, in the closed-loop game, however, capacities invested in the first stage and then they are sold in the second stage in a spot market. They find that market outcomes (investments and outputs) in the closed-loop game are in between the open-loop game and the efficient outcomes. Bushnell and Ishii [3] study a simulation model of a discrete-time dynamic Cournot game in which firms make lumpy investment decisions. They calculate the Markov perfect equilibrium investment levels in oligopoly. They find that uncertainty in demand growth can delay investment. Garcia and Shen [7] characterize Markov perfect equilibrium capacity expansion plans for oligopoly. They find that Cournot firms underinvests relative to the social optimum. Garcia and Stacchetti [8] study a dynamic Bertrand game with capacity constraints with random demand growth and periodic investments. They find that in some equilibria total capacity falls short of demand, and hence system security is jeopardized. They also find that price caps do not affect the optimum investment levels. These papers assume symmetric technologies with constant marginal cost of production. In our paper, we assume asymmetric technologies with different cost structures. This is an important feature in electricity generation industry, which is the focus of this paper. We also compare different behavioral strategies (Markov perfect versus open-loop) that might be used by power generators before making investment decisions.

We examine a two-period duopoly market with one firm operating a hydroelectric generating plant and another operating a thermal generating plant. Both firms have capacity constraints, but only the thermal producer is able to invest in increasing its capacity. In the first period both producers choose their outputs, and the thermal player also chooses investment level that will be productive in the following period. In the second period both players simultaneously choose their production levels given their capacities. Demand for electricity in the second period is stochastic due to uncertain demand growth. We analyze

the choice of capacity by the thermal producer under demand uncertainty and characterize both the Markov perfect and S-adapted open-loop equilibria under both binding and nonbinding hydro production constraints. We find that thermal investment is higher under Markov perfect information and this investment may be either higher or lower than the efficient. We also find that optimal investment is a discontinuous function of initial capacity under the Markov perfect equilibrium, and it is a continuous function under the open-loop structure.

\section{The model}

The firms compete over two periods, $t=0,1$. In period 0 , inverse demand is known to be $P_{0}(Q)=D-Q$, with $D$ a constant and $Q$ the total output of the two firms. Inverse 
demand in period 1 is random:

$$
P_{1}(Q)=\left\{\begin{array}{cc}
D+\bar{\delta}-Q & \text { with probability } u \\
D-\underline{\delta}-Q & \text { with probability } d
\end{array}\right.
$$

with $u+d=1$, and $\bar{\delta}>\underline{\delta} \geq 0$. This demand model allows different jump levels in demand intercept, including increasing or flat demand levels in period 1 . The expected demand in period 1 is $\delta(2 u-1)$, whenever $\bar{\delta}=\underline{\delta}=\delta$. Hence, the demand growth will be positive as long as $u>0.5$.

There are two types of technologies in the industry: a hydroelectric generator owns generation units that use water held behind dams to spin the electric generators and a thermal electric generator owns thermal units that burn fossil fuel to turn the turbine. These different generation technologies result in different cost functions for the two firms. Thermal generation is governed by the cost function $C(q)=c_{1} q+\left(c_{2} / 2\right) q^{2}$ for thermal output $(q)$ less than the thermal generator's capacity, which we denote by $K_{t}$ at time $t^{3}$ The thermal generator can expand capacity through investment in period 0 . An investment of $I_{0}$ units of capacity costs the thermal producer $\left(e_{1} / 2\right) I_{0}^{2}$. Investment is irreversible: $I_{0} \geq 0$ and capacity does not depreciate. The thermal producer begins the game with $K_{0}$ units of capacity, so in period one has $K_{1}=K_{0}+I_{0}$ units of capacity available. Actions taken by the thermal producer consist of investment and production in period 0 and production in each of the period 1 demand states: $\left(I_{0}, q_{0}, q_{1 u}, q_{1 d}\right){ }^{4}$

Hydroelectric power generation is generally thought of as having lower operating costs which we model by assuming that the marginal cost of production for hydro units is zero. ${ }^{5}$ In each period there is a maximal amount of hydro electricity that can be generated denoted by $W_{0}$. We think of this capacity as the carrying capacity of the reservoir. Essentially, we are assuming that there is sufficient inflow of water between periods 0 and 1 to restore $W_{0}$ by the beginning of period $1 .{ }^{6}$ As such, one could think of our hydro producer as any generator with a low marginal cost but fixed capacity (such as one with a nuclear generation technology). The hydro producer must choose three actions in this game: period 0 production and period 1 production in each of the two demand states. We denote a vector of hydro producer actions as $\left(h_{0}, h_{1 u}, h_{1 d}\right)$.

It will be useful in the presentation of the results to let $q_{u}^{c}$ and $q_{d}^{c}$ denote the Cournot equilibrium thermal outputs in the first period game when no constraints bind and $q_{0}^{c}$ the corresponding quantity in period 0 , i.e.,

$$
q_{0}^{c} \equiv \frac{D-2 c_{1}}{3+2 c_{2}}, \quad q_{d}^{c} \equiv \frac{D-\underline{\delta}-2 c_{1}}{3+2 c_{2}}, \quad q_{u}^{c} \equiv \frac{D+\bar{\delta}-2 c_{1}}{3+2 c_{2}} .
$$

\footnotetext{
${ }^{3}$ We do not model the transmission network, and hence assume away transmission constraints, transmission loss and congestion issues.

${ }^{4}$ We use the subscripts $1 u$ to denote period one with high demand and $1 d$ to denote period one with low demand.

${ }^{5}$ The marginal cost of production is generally assumed to be zero, since the water turning the turbines is commonly free.

${ }^{6}$ As the length of time between periods represents how long it takes to install additional thermal capacity, the assumption is that the reservoir refills "quickly" relative to the time to build capacity.
} 
The timing of the game is as follows. In period 0 , players choose production quantities simultaneously and independently to maximize their own profits. At the same time, the thermal producer chooses how much to invest in period 1 capacity. In the second period, players make their optimal production decisions conditional on the demand state that reveals under the open-loop structure, and they condition their decisions on the capacity and demand states under the Markov-perfect structure.

We next turn to analysis of the game when the hydro constraint is non-binding. Following that we examine a case with a binding hydro capacity constraint.

\section{Unconstrained hydro production}

In this section, we will present investment strategies for the S-adapted open-loop and the Markov perfect equilibria under the assumption that the initial water capacity, $W_{0}$, is sufficiently large that the production constraint will not be binding for the hydro generator.

\subsubsection{S-adapted open-loop equilibrium}

In this subsection, we wish to compute the equilibrium outcome when the thermal producer does not choose its investment level strategically. If there were no uncertainty, the appropriate equilibrium concept would be the open-loop Nash equilibrium. However, we want the producers to be able to respond to the future demand state, in which case the appropriate solution concept is the S-adapted open-loop equilibrium. Here we assume that players have S-adapted information. ${ }^{7}$ Under S-adapted information, the producers can adjust their period one strategies to the demand state but not to the level of thermal capacity, $K_{1}$. This means that there will be no strategic component to the thermal producer's investment decision.

In terms of our model, strategies can depend explicitly on the demand state, but not on the level of thermal capacity. An S-adapted strategy for the hydro producer is $\sigma^{H}=$ $\left(h_{0}, h_{1 u}, h_{1 d}\right)$, where $h_{1 u}$ is period one production in the high demand state and $h_{1 d}$ is period one production in the low demand state. The thermal producer's strategy is $\sigma^{T}=$ $\left(I_{0}, q_{0}, q_{1 u}, q_{1 d}\right)$. Each player chooses its own strategy to maximize its payoff function given the rival's strategy.

The hydro producer chooses its strategy to solve

$$
\max _{\sigma^{H}} E_{0} \sum_{t \in\{0,1 u, 1 d\}}\left(D_{t}-\left(h_{t}+q_{t}\right)\right) h_{t}
$$

subject to

$$
0 \leq h_{t} \leq W_{0}
$$

\footnotetext{
${ }^{7}$ This equilibrium concept first introduced by Haurie, Zaccour and Smeers [11]. It is extended and employed for large-scale oligopolies by Haurie and Moresino [12], Genc, Reynolds and Sen [9], and Genc and Sen [10]. In this equilibrium, players condition their decisions on time period, demand state and initial capacity. This equilibrium concept is between closed loop and open loop equilibrium concepts (see, e.g., Genc, Reynolds and Sen [9] and Pineau and Murto [15]).
} 
$E_{0}$ denotes the expectation taken with respect to information available at time 0 . As mentioned above, we assume that $W_{0}$ is sufficiently large that the capacity constraint will not be binding.

The thermal producer faces the problem:

$$
\max _{\sigma^{T}} E_{0} \sum_{t \in\{0,1 u, 1 d\}}\left[\left(D_{t}-\left(h_{t}+q_{t}\right)\right) q_{t}-c_{1} q_{t}-\frac{c_{2}}{2} q_{t}^{2}\right]-\frac{e_{1}}{2} I_{0}^{2}
$$

subject to

$$
\begin{gathered}
0 \leq q_{t} \leq K_{t}, \\
K_{1}=K_{0}+I_{0} .
\end{gathered}
$$

The following proposition summarizes the equilibrium strategies for this game:

Proposition 1:For $W_{0}$ sufficiently large, that is $W_{0}>\left(D+\bar{\delta}-K_{0}-I_{0 b}^{O}\right) / 2$, where $I_{0 b}^{O}$ is defined below, that the hydro producer is not constrained, the $S$ adapted open-loop Nash equilibrium investment strategies are:

$$
I_{0}^{O}=\left\{\begin{array}{cc}
0 & \text { if } q_{u}^{c}<K_{0} \\
\frac{u\left(D+\bar{\delta}-2 c_{1}-K_{0}\left(3+2 c_{2}\right)\right)}{2 e_{1}+u\left(3+2 c_{2}\right)}=I_{0 a}^{O} & \text { if } \underline{K}_{0}<K_{0}<q_{u}^{c} \\
\frac{\left(D-\underline{\delta}-2 c_{1}-K_{0}\left(3+2 c_{2}\right)\right)+u(\bar{\delta}+\underline{\delta})}{2 e_{1}+\left(3+2 c_{2}\right)}=I_{0 b}^{O} & \text { if } 0<K_{0}<\underline{K}_{0}
\end{array}\right.
$$

with

$$
\underline{K}_{0}=q_{d}^{c}-\frac{u(\bar{\delta}+\underline{\delta})}{2 e_{1}} .
$$

Proof: Since there is enough water available that the hydro constraints do not bind, the hydro producer plays its "static" best response in each period. Specifically $h_{0}=\frac{D-q_{0}}{2}, h_{1 u}=\frac{D+\bar{\delta}-q_{1 u}}{2}$, and $h_{1 d}=\frac{D-\frac{\delta}{2}-q_{1 d}}{2}$.

The period 0 thermal production choice has no bearing on the payoffs of any of other thermal actions, so which case obtained below is of no consequence to the equilibrium investment and period 1 outputs. The Lagrangian function for the thermal producer's problem is

$$
\begin{array}{r}
L^{T}=E_{0} \sum_{t \in\{0,1 u, 1 d\}}\left[\left(D_{t}-\left(h_{t}+q_{t}\right)\right) q_{t}-c_{1} q_{t}-\frac{c_{2}}{2} q_{t}^{2}\right]-\frac{e_{1}}{2} I_{0}^{2} \\
+\sum_{t \in\{0,1 u, 1 d\}}\left[a_{t}\left(K_{t}-q_{t}\right)\right]
\end{array}
$$

where $a_{t} \geq 0$ are the Lagrange multipliers on the capacity constraints ${ }^{8}$. The $K K T$ conditions for the thermal producer's problem are then

$$
\frac{\partial L^{T}}{\partial q_{t}} q_{t}=0, \quad \frac{\partial L^{T}}{\partial a_{t}} a_{t}=0 \quad \text { and } \frac{\partial L^{T}}{\partial I_{0}} I_{0}=0
$$

\footnotetext{
${ }^{8}$ The non-negativity constraints do not bind for the situations we are interested in, so we suppress their multipliers to simplify the presentation.
} 
for $t=0,1 u, 1 d$.

When the initial capacity is high enough, that is it is greater than the Cournot output in the high demand state, $q_{u}^{c}<K_{0}$, investment is clearly zero. Investment will not be used and it is costly, hence it must be zero in equilibrium.

Given the assumption $\underline{K}_{0}<K_{0}<q_{u}^{c}$, it is clear that $a_{1 u}>a_{1 d}=0$. It follows that $q_{1 u}=K_{0}+I_{0}$, and $q_{1 d}=\frac{D-\underline{\delta}-h_{1 d}-c_{1}}{2+c_{2}}$. The output levels at $t=0$ is irrelevant of investment decision because of the lag between investment and production, hence either $q_{0}=\frac{D-h_{0}-c_{1}}{2+c_{2}}$, or $q_{0}=K_{0}$ holds.

Next we solve the best response functions for the equilibrium points. By substituting one player's response functions into other's functions we obtain that $q_{1 d}=$ $\frac{(D-\underline{\delta})-2 c_{1}}{3+2 c_{2}}$ and $h_{1 d}=\frac{(D-\underline{\delta})\left(1+c_{2}\right)+c_{1}}{3+2 c_{2}}$. Since $q_{1 u}=K_{0}+I_{0}, h_{1 u}=\frac{D+\bar{\delta}-K_{0}-I_{0}}{2}$. At time 0, either $q_{0}=\frac{D-2 c_{1}}{3+2 c_{2}}$ or $q_{0}=K_{0}$ and the hydro producer plays its best response.

For optimal investment outcomes we note that the period one capacity constraints only bind when demand is high, so investment only has an impact in that state. We then obtain $a_{1 u}=u\left[D+\bar{\delta}-2 q_{1 u}-h_{1 u}-c_{2} q_{1 u}-c_{1}\right]$. Using the equilibrium $q_{1 u}$ and $h_{1 u}$ from above and noting that the optimal investment choice satisfies $I_{0}=\frac{a_{1 u}}{e_{1}}$, we get the equilibrium $I_{0}$ of the proposition.

Next, given the assumption $0<K_{0}<\underline{K}_{0}$, it is clear that $a_{1 u}>0, a_{1 d}>0$. It follows that $q_{1 u}=K_{0}+I_{0}=q_{1 d}$. Next we solve the best response functions for the equilibrium points. By substituting one player's response functions into other's functions we obtain that $h_{1 d}=\frac{D-\underline{\underline{\delta}-K_{0}-I_{0}}}{2}$, since $q_{1 d}=K_{0}+I_{0}$. Also, since $q_{1 u}=K_{0}+I_{0}, h_{1 u}=\frac{D+\bar{\delta}-K_{0}-I_{0}}{2}$. At time 0, either $q_{0}=\frac{D-2 c_{1}}{3+2 c_{2}}$ or $q_{0}=K_{0}$ and the hydro producer plays its best response.

For optimal investment outcomes we note that the period one capacity constraints bind in both demand states. We then obtain $a_{1 u}=u\left[D+\bar{\delta}-2 q_{1 u}-h_{1 u}-\right.$ $\left.c_{2} q_{1 u}-c_{1}\right], a_{1 d}=(1-u)\left[D-\underline{\delta}-2 q_{1 d}-h_{1 d}-c_{2} q_{1 d}-c_{1}\right]$. Using the equilibrium $q_{1 u}, q_{1 d}, h_{1 u}$ and $h_{1 d}$ from above and noting that the optimal investment choice satisfies $I_{0}=\frac{a_{1 u}+a_{1 d}}{e_{1}}$, we get the equilibrium $I_{0}$ of the proposition.

We obtain the lower bound of initial capacity, $\underline{K}_{0}$, that entails non-binding capacity at the down state demand by solving $K_{0}+I_{0} \geqslant q_{d}^{c}$. Similarly, we obtain the upper bound of the initial capacity that entails binding capacity at the down state demand by solving $K_{0}+I_{0}<q_{d}^{c}$, where the different investment expressions are as defined in the proposition. Because of the continuity of investments the upper bound and the lower bound on the initial capacities are identical. In this proposition we assume that the water level is plentiful enough so that water constraints do not bind at all. We need to find the lower bound of the water level in which water constraints do not bind at any period. Hydro best response in the upstate demand is $h_{1 u}=\left(D+\bar{\delta}-q_{1 u}\right) / 2$. The maximum value of this best response, denoted by $\bar{h}_{1 u}$, is obtained when $q_{1 u}$ gets its minimum value. This 
happens when $q_{1 u}=K_{0}+I_{0}$, in which $I_{0}=I_{0 b}^{O}$ satisfying (5) for the interval $0<K_{0}<\underline{K}_{0}$. Therefore, whenever $W_{0}>\bar{h}_{1 u}$ is satisfied the equilibrium proposed in proposition 1 characterizes investment levels for the case in which water level is high and water constraints are interior for all demand levels. $\square$

When there is substantial initial thermal capacity, the initial capacity is enough to cover the maximum Cournot output, $K_{0}>q_{u}^{c}$, the equilibrium investment must be zero. Any incremental investment would generate excess non-utilized capacity at a positive cost. Hence, the optimum investment strategy by the thermal player is "do not invest". For low initial capacity, $0<K_{0}<\underline{K}_{0}$, the period 1 capacity binds in both demand states. In this case, the level of investment is determined by the sum of the capacity prices (i.e., shadow prices for the binding capacity constraints). For intermediate values of initial thermal capacity, $\underline{K}_{0}<K_{0}<q_{u}^{c}$, the period 1 capacity constraint is binding if demand is high and non-binding if demand is low. In this case, only the shadow price of capacity in the high demand influences investment. Capacity will be fully utilized in the high demand state and there will be excess capacity in the low demand state.

The comparative statics on investment following from Proposition 1 are natural. Equilibrium investment is increasing in the probability of high demand $(u)$ and the level of demand $(D)$ and decreasing in initial capacity $\left(K_{0}\right)$, the cost of investment $\left(e_{1}\right)$, and the cost of thermal production $\left(c_{1}\right.$ and $\left.c_{2}\right)$.

In order to discuss the implications of strategic behavior on investment we next analyze equilibrium investment under Markov perfect information.

\subsubsection{Markov Perfect Equilibrium}

In the S-adapted open-loop equilibrium, the thermal producer does not take into account the influence that its investment choice has on the hydro producer's output choice in period one. This is a consequence of the S-adapted information structure. Players using open-loop strategies commit to their strategies at the beginning of the game, that is each player's choice of actions is predetermined. However Markovian strategies are state dependent and under which players do not commit to their action plans at the outset. Denote the Markov perfect strategies of the two producers by $\sigma^{H}\left(D_{t}, W_{t}\right)$ and $\sigma^{T}\left(D_{t}, W_{t}\right)$. We assume that both producers observe $W_{t}$ and $D_{t}$ before making decisions in period $t$. The Markov perfect equilibrium is a Nash equilibrium in Markov perfect strategies.

Proposition 2: For $W_{0}$ sufficiently large, that is $W_{0}>\left(D+\bar{\delta}-K_{0}-I_{0 b}^{M}\right) / 2$, where $I_{0 b}^{M}$ is defined below, that the hydro producer is not constrained, the Markov perfect Nash equilibrium investment strategies are:

$$
I_{0}^{M}=\left\{\begin{array}{cc}
0 & \text { if } q_{u}^{c}<K_{0} \\
\frac{u\left(D+\bar{\delta}-2 c_{1}-K_{0}\left(2+2 c_{2}\right)\right)}{2 e_{1}+u\left(2+2 c_{2}\right)}=I_{0 a}^{M} & \text { if } \underline{K}_{0}^{\prime}<K_{0}<q_{u}^{c} \\
-K_{0}+q_{d}^{c} & \text { if } \underline{K}_{0}^{\prime \prime}<K_{0}<\underline{K}_{0}^{\prime} \\
\frac{\left(D-\underline{\delta}-2 c_{1}-K_{0}\left(2+2 c_{2}\right)\right)+u(\bar{\delta}+\underline{\delta})}{2 e_{1}+\left(2+2 c_{2}\right)}=I_{0 b}^{M} & \text { if } 0<K_{0}<\underline{K}_{0}^{\prime \prime}
\end{array}\right.
$$


where

$$
\underline{K}_{0}^{\prime}=q_{d}^{c}\left(1-\frac{u}{2 e_{1}}\right)-\frac{u(\bar{\delta}+\underline{\delta})}{2 e_{1}}
$$

and

$$
\underline{K}_{0}^{\prime \prime}=q_{d}^{c}\left(1-\frac{1}{2 e_{1}}\right)-\frac{u(\bar{\delta}+\underline{\delta})}{2 e_{1}} .
$$

Proof: The only difference in the proof of this proposition and that of Proposition 1 is in the determination of investment. The best responses by both players in period one are the same as they are in the S-adapted open-loop game. Hence, conditional on $K_{1}$, outputs in period one are the same. However, investment in capacity by the thermal producer, and hence $K_{1}$, may differ.

Under the assumption $\underline{K}_{0}^{\prime}<K_{0}<q_{u}^{c}$, investment only provides benefits in stage $1 u$. Let $\pi_{1 u}^{T}\left(K_{1}\right)$ be the profit to the thermal investor in period $1 u$ when it has capacity of $K_{1}=K_{0}+I_{0}$. Optimal investment must satisfy

$$
-e_{1} I_{0}+u \frac{\partial \pi_{1 u}^{T}}{\partial K_{1}} \frac{\partial K_{1}}{\partial I_{0}}=0
$$

or

$$
-e_{1} I_{0}+u\left[D+\bar{\delta}-h_{1 u}\left(K_{1}\right)-2 K_{1}-K_{1} h_{1 u}^{\prime}\left(K_{1}\right)-c_{1}-c_{2} K_{1}\right]=0
$$

When $q_{1 u}=K_{1}$, we know that $h_{1 u}\left(K_{1}\right)=\frac{D+\bar{\delta}-K_{1}}{2}$ is the hydro producers best response. Substituting this for $h_{1 u}\left(K_{1}\right)$ and $K_{1}=K_{0}+I_{0}$ and simplifying we have

$$
I_{0 a}=\frac{u\left[D+\bar{\delta}-K_{0}\left(2+2 c_{2}\right)-2 c_{1}\right]}{2\left(e_{1}+u\left(1+c_{2}\right)\right)}
$$

We obtain the lower bound of initial capacity, $\underline{K}_{0}^{\prime}$, that entails non-binding capacity at the down state demand by solving $K_{0}+I_{0 a} \geqslant q_{d}^{c}$.

Under the assumption $0<K_{0}<\underline{K}_{0}^{\prime \prime}$, investment provides benefits in both stages $1 u$ and $1 d$. The optimal investment must satisfy

$$
\begin{aligned}
& -e_{1} I_{0}+u\left[D+\bar{\delta}-h_{1 u}\left(K_{1}\right)-2 K_{1}-K_{1} h_{1 u}^{\prime}\left(K_{1}\right)-c_{1}-c_{2} K_{1}\right] \\
& \quad+(1-u)\left[D-\underline{\delta}-h_{1 d}\left(K_{1}\right)-2 K_{1}-K_{1} h_{1 d}^{\prime}\left(K_{1}\right)-c_{1}-c_{2} K_{1}\right]=0 .
\end{aligned}
$$

When $q_{1 u}=K_{1}, h_{1 u}\left(K_{1}\right)=\frac{D+\bar{\delta}-K_{1}}{2}$ is the hydro producer's best response, and when $q_{1 d}=K_{1}, h_{1 d}\left(K_{1}\right)=\frac{D-\frac{\delta}{2} K_{1}}{2}$. Substituting these terms into the above optimality condition and simplifying we obtain

$$
I_{0 b}=\frac{\left[D-\underline{\delta}-K_{0}\left(2+2 c_{2}\right)-2 c_{1}\right]+u[\bar{\delta}+\underline{\delta}]}{2\left(e_{1}+1+c_{2}\right)}
$$

Also, we obtain the upper bound of initial capacity, $\underline{K}_{0}^{\prime \prime}$, that entails binding capacity at the down state demand by solving $K_{0}+I_{0 b}<q_{d}^{c}$. 
The remaining capacity interval is $\underline{K}_{0}^{\prime \prime}<K_{0}<\underline{K}_{0}^{\prime}$. When the initial capacity falls into this region in which $\underline{K}_{0}^{\prime}<q_{d}^{c}$, the optimal investment will satisfy $I_{o}=q_{d}^{c}-K_{0}$ so that period 1 capacity just becomes equal to the Cournot output in the downstate demand. In this proposition we assume that the water level is "high" so that water constraints do not bind at all. The water level that satisfies this property is calculated as follows. The hydro best response in the upstate demand is $h_{1 u}=\left(D+\bar{\delta}-q_{1 u}\right) / 2$. The maximum value of this best response, denote $\bar{h}_{1 u}$, is obtained when $q_{1 u}$ gets its minimum value. This happens when $q_{1 u}=K_{0}+I_{0 b}^{M}$. Therefore, whenever $W_{0}>\bar{h}_{1 u}$ is satisfied the equilibrium proposed in proposition 2 characterizes investment levels for the case in which water level is high and water constraints are interior for all demand stages.

The investment rule defined in Proposition 2 clearly satisfies $I_{0 a}^{M}<-K_{0}+q_{d}^{c}<I_{0 b}^{M}$. The investment $I_{0 a}^{M}$ is obtained when the thermal production constraint binds in the high demand state only. Whereas, $I_{0 b}^{M}$ is obtained when the thermal production constraints bind in both high and low demand states. Both $I_{0 a}^{M}$ and $I_{0 b}^{M}$ are decreasing in the investment cost parameter $e_{1}$. However, the investment level is unaffected by the investment cost parameter in some cases (when it is equal to $-K_{0}+q_{d}^{c}$ ). The reason for this is that the marginal profit of investment is discontinuous at the investment level of $-K_{0}+q_{d}^{c}$. At this level, $K_{1}$ is equal to the Cournot output at low demand, $q_{d}^{c}$. At an investment level lower than $-K_{0}+q_{d}^{c}$ the capacity constraint for the thermal producer will bind if demand is low. In this case, the marginal profit of investment includes the benefit of additional capacity in both high and low demand states. However, for investment larger than $-K_{0}+q_{d}^{c}$ the marginal profit of investment includes the benefit of additional capacity in the high demand state alone, as the benefit is nil in the low demand state. Consequently the marginal profit of investment has a downward-jumping discontinuity at $I_{0}=-K_{0}+q_{d}^{c}$, and when $K_{0} \in\left(\underline{K}_{0}^{\prime \prime}, \underline{K}_{0}^{\prime}\right)$ marginal profit is positive to the left of this discontinuity and negative to the right. This results in the thermal producer choosing to invest the precise quantity that yields $K_{1}=q_{d}^{c}$.

A second feature of note in the investment rule of Proposition 2 is that it is discontinuous, since

$$
\lim _{K_{0} \rightarrow q_{u}^{c-}} I_{0}^{M}>0
$$

and

$$
\lim _{K_{0} \rightarrow q_{u}^{c+}} I_{0}^{M}=0 .
$$

For $K_{0}>q_{u}^{c}$ investment will yield no benefit since the capacity constraint will not bind in the high demand state, whereas, for $K_{0}<q_{u}^{c}$ investment yields a strictly positive benefit in period 1 since the capacity constraint binds with a positive probability.

The two discontinuities in the the marginal profit of investment for the thermal producer are a result of the thermal producer taking into account how its capacity in period 1 affects the hydro producer's output. The hydro producer's output as a function of $K_{1}$ is not smooth across the two thresholds generating the discontinuities. Hence the investment rule in the S-adapted equilibrium of Proposition 1 does not exhibit the same behavior as that 
in the Markov perfect equilibrium.

The strategic behavior of the thermal producer also has implications for the level of investment. We know from the proof of Proposition $2, \partial h_{1 u} / \partial I_{0}^{M}=-1 / 2$ in the Markov perfect game, which should lead to higher equilibrium investment, i.e., the strategic effect associated with investment in thermal capacity results in "aggressive" behavior by the thermal producer. We summarize this result in:

Corollary: The Markov perfect equilibrium investment is larger than the $S$ adapted open-loop equilibrium investment.

Proof: We will give the sketch of the proof, since we described the rationale above. When we closely look at the investment expressions under Markov perfect and open-loop equilibria we notice four regions of initial capacity under which we should compare investment expressions. The first region is $R_{1}^{O}$ and found as follows. Let $R_{1}^{O}=\left\{K_{0} \mid \underline{K}_{0}<K_{0}<q_{u}^{c}\right\}$, and $R_{1}^{M}=\left\{K_{0} \mid \underline{K}_{0}^{\prime}<K_{0}<q_{u}^{c}\right\}$. Clearly $R_{1}^{O} \cap R_{1}^{M}=R_{1}^{O}$ since $\underline{K}_{0}>\underline{K}_{0}^{\prime}$. Under the region $R_{1}^{O}$, clearly $I_{0 a}^{O}<I_{0 a}^{M}$. Let the second region be $R_{2}^{M}$, which is computed as follows. $R_{2}^{O}=\left\{K_{0} \mid 0<\right.$ $\left.K_{0}<\underline{K}_{0}\right\}$, and $R_{2}^{M}=\left\{K_{0} \mid 0<K_{0}<\underline{K}_{0}^{\prime \prime}\right\}$, and $R_{2}^{O} \cap R_{2}^{M}=R_{2}^{M}$ since $\underline{K}_{0}>\underline{K}_{0}^{\prime \prime}$. Under the region $R_{2}^{M}$, clearly $I_{0 b}^{O}<I_{0 b}^{M}$.

The third region is denoted by $R_{3}^{M}$, which is the intersection of $R_{2}^{O}$ and $R_{3}^{M}$, where $R_{3}^{M}=\left\{K_{0} \mid \underline{K}_{0}^{\prime \prime}<K_{0}<\underline{K}_{0}^{\prime}\right\}$ and the bounds on the initial capacities are as defined above in the text. The investment expressions under the region $R_{3}^{M}$ are $I_{0 b}^{O}$ and $I_{0 c}^{M}=-K_{0}+q_{d}^{c}$ for open-loop and Markov perfect structures, respectively. After substituting $I_{0 c}^{M}$ into $I_{0 b}^{O}$ and rearranging the terms, we obtain

$$
I_{0 b}^{O}=\frac{I_{0 c}^{M}\left(3+2 c_{2}\right)}{2 e_{1}+3+2 c_{2}}+\frac{u(\bar{\delta}+\underline{\delta})}{2 e_{1}+3+2 c_{2}}
$$

Note that to have the region $R_{3}^{M}$ exist we need to have $\underline{K}_{0}^{\prime \prime}>0$. This implies $q_{d}^{c}\left(1-\frac{1}{2 e_{1}}\right)>\frac{u(\bar{\delta}+\underline{\delta})}{2 e_{1}}$ and also $e_{1}>1 / 2$. Given these conditions and the above equality we obtain that $I_{0 b}^{O}<I_{0 c}^{M}$.

The fourth region for the initial capacity is as a result of intersection of $R_{2}^{O}$ and $R_{1}^{M}$. Denote this intersection $R_{4}^{M}$, and $R_{4}^{M}=\left\{K_{0} \mid \underline{K}_{0}^{\prime}<K_{0}<\underline{K}_{0}\right\}$. In this region we need to compare $I_{0 a}^{M}$ and $I_{0 b}^{O}$. Given that to have this region exist we need to satisfy $\underline{K}_{0}^{\prime}>0$ and $\underline{K}_{0}<q_{d}^{c}$, we find that $I_{0 a}^{M}>I_{0 b}^{O}$.

To illustrate investment patterns under both equilibrium concepts we solve the model for an example. We employ the following parameters and compute the optimal investments as a function of initial capacity $K_{0}$, as the initial capacity varies in the regions specified in the propositions 1 and 2 . We use $D=100, u=0.5, \bar{\delta}=10, \underline{\delta}=0, c_{1}=0, c_{2}=1, e_{1}=1$.

In Figure 1 the vertical axis denotes investment and the horizontal axis denotes initial capacity. The equilibrium investment under open-loop structure is represented by a solid line in which there are three regimes as a result of three regions for initial capacities. As 


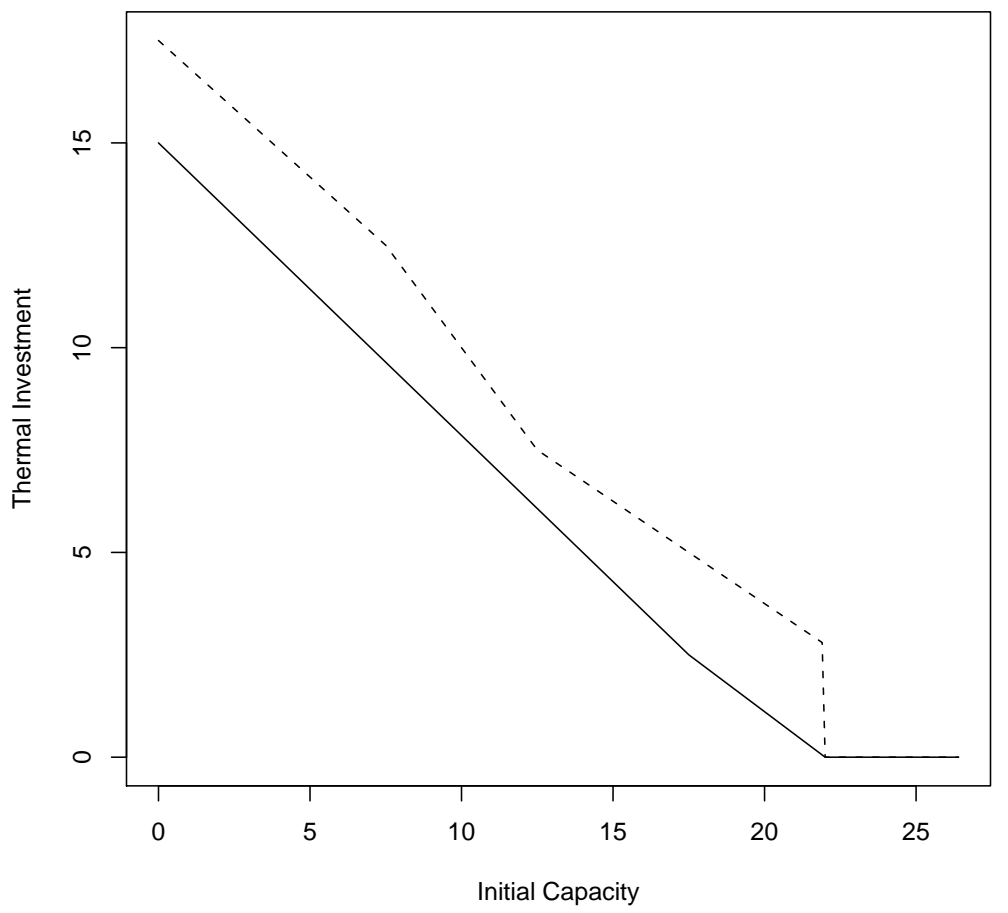

Figure 1: Thermal investment: S-adapted Open Loop (solid line) and Markov Perfect (dashed line)

computed in proposition 1 in equation (5), the slope for equilibrium investment starts from $-\left(3+2 c_{2}\right) /\left(2 e_{1}+3+2 c_{2}\right)=-5 / 7$ then drops (in absolute value) to $-u\left(3+2 c_{2}\right) /\left(2 e_{1}+u(3+\right.$ $\left.\left.2 c_{2}\right)\right)=-5 / 9$ and finally reaches to zero in which investment quantity is zero since initial capacity is abundant. The dashed line represents Markov perfect equilibrium investment as a function of the initial capacity. There are four different slopes for the investment function under Markov perfect equilibrium and the investment function is discontinuous. It starts from $-\left(2+2 c_{2}\right) /\left(2 e_{1}+2+2 c_{2}\right)=-2 / 3$ and increases (in absolute value) to -1 and then drops to $-u\left(2+2 c_{2}\right) /\left(2 e_{1}+u\left(2+2 c_{2}\right)\right)=-1 / 2$ and finally jumps to zero. The jump in investment function happens when there is enough capacity to cover the Cournot output in the highest demand scenario. The marginal profit of thermal player in investment drops below zero since the cost of each incremental investment exceeds the benefit of that investment. Indeed there is no benefit of each incremental investment when hydro player production is unconstrained and thermal initial capacity is greater than the maximum Cournot output. Also, as can be seen in the figure investment under Markov perfect information structure exceeds that of under open-loop structure.

In Figure 2, we plot $K_{1}$ versus $K_{0}$ given the equilibrium investments in Figure 1 . This figure has the same characteristics as that in Figure 1. Period 1 capacities become identical when there is no investment under both equilibria, otherwise Markov perfect equilibrium market capacity exceeds that of under the open-loop one. 




Figure 2: Thermal capacity: S-adapted Open Loop (solid line) and Markov Perfect (dashed line)

\section{Constrained hydro production}

In the previous section, we examined optimum thermal investment behavior when the hydro player had plenty of water in which its production constraint did not bind at all. Now we relax this assumption and allow binding water constraints in both periods of the game. There are two possibilities here: $W_{0}$ can be very low such that the hydro constraint binds in both periods, or of an intermediate level where it binds in the high demand state only. We focus on the former case here. ${ }^{9}$ This case represents a market structure in which the thermal player faces a small hydro player that does not have enough reservoir capacity to satisfy its Cournot output in the low demand scenario. The thermal firm will act like a monopolist facing hydro player whose supply is perfectly inelastic.

When the water level is low (i.e., $0<W_{0}<h_{1 d}^{c}$ ) the water constraint binds in both high and low demand cases and the thermal player acts as a monopolist facing the residual demand given by $q_{1 u}=D+\bar{\delta}-W_{0}-P_{1 u}$ and $q_{1 d}=D-\underline{\delta}-W_{0}-P_{1 d}$, where $P_{1 u}$ and $P_{1 d}$ are market prices at up and down states in period $1 .{ }^{10}$ In this case the S-adapted open-loop

\footnotetext{
${ }^{9}$ We have analyzed the latter case in which water constraint only binds in the high demand state, however the investment behavior is qualitatively similar to that in Section 3.1, hence we do not report the results here.

${ }^{10}$ Indeed, the tightest upper bound for the initial water level under which hydro constraint is always binding satisfies $W_{0}<h_{1 d}=\left(D-\underline{\delta}-q_{d}\right) / 2$, where $q_{d}=K_{0}+I_{0}$ and $I_{0}$ is defined above in expression (16) for the initial capacity interval $0<K_{0}<\underline{K}_{0}$.
} 
Nash equilibrium investment strategies will satisfy:

$$
I_{0}=\left\{\begin{array}{cc}
0 & \text { if } \bar{q}_{u}<K_{0} \\
\frac{u\left[D+\bar{\delta}-c_{1}-K_{0}\left(2+c_{2}\right)-W_{0}\right]}{e_{1}+u\left(2+c_{2}\right)} & \text { if } \underline{K}_{0}<K_{0}<\bar{q}_{u} \\
\frac{\left[D-\underline{\delta}-c_{1}-K_{0}\left(2+c_{2}\right)-W_{0}\right]+u[\bar{\delta}+\underline{\delta}]}{e_{1}+2+c_{2}} & \text { if } 0<K_{0}<\underline{K}_{0}
\end{array}\right.
$$

For the sake of briefness we do not write all the steps that give directions to the solution, but we describe how we obtain the above equilibrium investment levels. In the case of constrained hydro production, there are three parameter regions in which i) either initial thermal capacity is high so that thermal production is unconstrained in the upstate and hence the investment quantity is zero, ii) or initial capacity is in medium level so that thermal player is constrained in the up demand state but unconstrained in the down demand state, and hence investment is positive to cover the demand in upstate, iii) or the initial thermal capacity is low so that the thermal player is constrained in both up and down demand states, in which the investment is positive and is being affected by both demand states.

When $\underline{K}_{0}<K_{0}<\bar{q}_{u}$ the best response functions will satisfy $q_{1 u}=K_{0}+I_{0}, h_{1 u}=W_{0}$, $q_{1 d}<q_{1 u}$, and $h_{1 d}=W_{0}$ in period 1 up and down states for both players. The optimum investment satisfies $I_{0}=a_{1 u} / e_{1}$, in which $a_{1 u}=u\left[D+\bar{\delta}-2 q_{1 u}-h_{1 u}-c_{2} q_{1 u}-c_{1}\right]$. Solving it for investment yields the above equilibrium level. When $0<K_{0}<\underline{K}_{0}$, the equilibrium output levels are $q_{1 u}=K_{0}+I_{0}=q_{1 d}, h_{1 u}=W_{0}=h_{1 d}$, the equilibrium investment satisfies $I_{0}=\left(a_{1 u}+a_{1 d}\right) / e_{1}$, in which $a_{1 u}=u\left[D+\bar{\delta}-2 q_{1 u}-h_{1 u}-c_{2} q_{1 u}-c_{1}\right]$ and $a_{1 d}=(1-u)\left[D-\underline{\delta}-2 q_{1 d}-h_{1 d}-c_{2} q_{1 d}-c_{1}\right]$. Solving it for the investment gives the above result. Clearly, when the initial capacity is high, that is $\bar{q}_{u}<K_{0}$, the thermal player does not invest. Note that the lower bound of initial capacity under which the thermal player does not invest satisfies $\bar{q}_{u}=\left(D+\bar{\delta}-c_{1}-W_{0}\right) /\left(2+c_{2}\right)$, which is the best response function of thermal player in the upstate demand when the rival hydro player dumps all of its available capacity into the market.

Next we characterize investment under the Markov perfect equilibrium when the water level is low. In this case the Markov perfect Nash equilibrium investment strategies are similar to the one described in Proposition 2 and will satisfy:

$$
I_{0}=\left\{\begin{array}{cc}
0 & \text { if } \bar{q}_{u}<K_{0} \\
\frac{u\left[D+\bar{\delta}-c_{1}-K_{0}\left(2+c_{2}\right)-W_{0}\right]}{e_{1}+u\left(2+c_{2}\right)} & \text { if } \underline{K}_{0}^{\prime}<K_{0}<\bar{q}_{u} \\
-K_{0}+q_{d}^{c} & \text { if } \underline{K}_{0}^{\prime \prime}<K_{0}<\underline{K}_{0}^{\prime} \\
\frac{\left[D-\underline{\delta}-c_{1}-K_{0}\left(2+c_{2}\right)-W_{0}\right]+u[\bar{\delta}+\underline{\delta}]}{e_{1}+2+c_{2}} & \text { if } 0<K_{0}<\underline{K}_{0}^{\prime \prime}
\end{array}\right.
$$

We calculate the equilibrium investments under the Markov perfect structure as follows.

1. In period 1 at the upstate demand the thermal best response function is $q_{1 u}=(D+$ $\left.\bar{\delta}-c_{1}-h_{1 u}\right) /\left(2+c_{2}\right)$. Since the water level is low the hydro player will produce at the available water level, which is $W_{0}$, then the best response quantity for thermal player becomes $q_{1 u}=\left(D+\delta-c_{1}-W_{0}\right) /\left(2+c_{2}\right)$. Denote this quantity $\bar{q}_{u}$. Clearly 
when $\bar{q}_{u}<K_{0}$ is satisfied upstate capacity constraint is non-binding and hence the investment quantity is zero.

2. When only upstate thermal constraint is binding the optimum investment satisfies $-e_{1} I_{0}+u\left[D+\bar{\delta}-h_{1 u}\left(K_{1}\right)-2 K_{1}-K_{1} h_{1 u}^{\prime}\left(K_{1}\right)-c_{1}-c_{2} K_{1}\right]=0$. The equilibrium outputs in the upstate become $h_{1 u}=W_{0}$ and $q_{1 u}=K_{0}+I_{0}$, and $h_{1 u}^{\prime}\left(K_{1}\right)=0$. Plugging these quantities into the above equality and simplifying, we obtain the optimal investment described above for $\underline{K}_{0}<K_{0}<\bar{q}_{u}$. The value of lower bound $\underline{K}_{0}$ can be obtained similar to the one obtained in proposition 2 : it will satisfy the property that downstate production is interior.

3. When the initial thermal capacity is "low", that is $0<K_{0}<\underline{K}_{0}^{\prime \prime}$, both upstate and downstate thermal production constraints do bind. In that case optimum investment satisfies,

$$
\begin{gathered}
-e_{1} I_{0}+u\left[D+\bar{\delta}-h_{1 u}\left(K_{1}\right)-2 K_{1}-K_{1} h_{1 u}^{\prime}\left(K_{1}\right)-c_{1}-c_{2} K_{1}\right] \\
(1-u)\left[D-\underline{\delta}-h_{1 d}\left(K_{1}\right)-2 K_{1}-K_{1} h_{1 d}^{\prime}\left(K_{1}\right)-c_{1}-c_{2} K_{1}\right]=0
\end{gathered}
$$

in which the optimum outputs are $h_{1 u}=W_{0}, q_{1 u}=K_{0}+I_{0}=q_{1 d}$, and $h_{1 d}=W_{0}$, and also $h_{1 u}^{\prime}\left(K_{1}\right)=0$ and $h_{1 d}^{\prime}\left(K_{1}\right)=0$. Plugging these expressions into the above expression we obtain the investment level for $0<K_{0}<\underline{K}_{0}^{\prime \prime}$. The bound $\underline{K}_{0}^{\prime \prime}$ can be obtained similar to the one obtained in proposition 2 : it will be equal to down state interior Cournot thermal output minus the above investment level for the interval $0<K_{0}<\underline{K}_{0}^{\prime \prime}$.

4. When the initial capacity satisfies $\underline{K}_{0}^{\prime \prime}<K_{0}<\underline{K}_{0}^{\prime}<q_{d}^{c}$, the optimal investment will satisfy $I_{o}=q_{d}^{c}-K_{0}$.

The comparison of the investment expressions (16) and (17) yields the following results. Whenever the water level is low so that hydro production is constrained all times, there are thermal capacity regions in which Markov perfect and S-adapted open-loop equilibria investment levels coincide. However, there are also regions where they do not coincide. Similar to the case with unconstrained hydro production, thermal investment is discontinuous in the Markov perfect equilibrium while it is continuous in the open-loop equilibrium. Finally, in the Markov perfect equilibrium there is a capacity region in which the thermal investment is independent of the hydro producer's capacity and output. Outside of this region there is a negative relationship between thermal investment and the hydro capacity.

\subsection{Discussion}

The results of the previous two sections imply that total output is higher in the Markov perfect equilibrium than in the open-loop equilibrium (when they differ). Consequently, prices are lower when strategic effects are allowed for. It is important to note that even 
though prices are lower, this does not mean that the Markov perfect equilibrium is necessarily more efficient. The increased output comes about through inefficient investment in the cases where the hydro producer has excess capacity. When the hydro producer is not operating at capacity clearly it would be efficient to increase output by increasing hydro production. In the Markov perfect equilibrium however, the increased output comes about through increased thermal production that is made possible through a costly investment.

Whether or not the thermal producer chooses a capacity that is greater or less than the efficient level of investment depends on two conflicting forces. First, since the hydro producer is restricting output, increasing thermal capacity can be efficient in that it reduces the loss due to the exercise of market power. Second, since the hydro producer has lower production costs, if it is not constrained it is efficient for any increased output to come from the hydro producer, not by the thermal producer increasing capacity. Either of these forces might dominate, hence we conclude that equilibrium capacity investment by the thermal producer may be either higher or lower than the efficient. To prove it we simply look at two limiting cases $W_{0} \rightarrow 0$ and $W_{0} \rightarrow \infty$. As $W_{0} \rightarrow 0$, we are in the setting of Section 4 . Hydro production goes to zero and the thermal producer has a monopoly. Thermal monopoly output and investment is clearly lower than what would be efficient. At the other extreme, as $W_{0} \rightarrow \infty$, equilibrium investment under duopoly is as we have described in Propositions 1 and 2 of Section 3, i.e., positive. In this case, the efficient level of investment is clearly zero since hydro production can meet all contingencies and we have over-investment relative to the efficient level.

\section{Conclusions}

Capacity investments in electricity markets is one of the main issues in the restructuring process to ensure competition and enhance system security of networks. In the presence of evolving demand, possible supply disruptions, and government incentives, production capacity investments have occurred in many jurisdictions. In this case a simple but an interesting question arises: What is the investment behavior when two different technologies compete in an electricity market? In this regard we have analyzed a duopolistic electricity market in which hydro and thermal generators compete when two different information structures may be observed.

We have studied dynamic competition between thermal and hydroelectric producers under demand uncertainty, showing that strategic effects result in higher investment in thermal generating capacity than when open-loop strategies are used. In addition, investment is a discontinuous function of initial capacity in the Markov perfect equilibrium. However, this higher investment may be inefficient. Essentially there are two sources of inefficiency: the distortion caused by market power and the distortion caused by the industry using an inefficient mix of generating technologies.

In our analysis, in the case of large and unconstrained hydro the thermal investment may be considered as optimum capacity chosen by entry of potential thermal producer. Indeed, in hydroelectricity dominated jurisdictions (like Quebec, Norway, Brazil, New Zealand) 
possible entry is expected by less capital intensive thermal generators. In this case, our paper presents optimal thermal investment under different behavioral assumptions. In the case of constrained hydro, the capacity expansion could be expected from thermal and nuclear generators. For instance, in Ontario, Canada hydro facilities are limited due to environmental and geographic constraints, and capacity investments are done by thermal generators.

In this paper we assumed a duopolistic market structure. Even though the quadratic thermal cost structure allows aggregate costs by different thermal generators, for the sake of simplicity we assumed the same ownership of these thermal generators. Also, we only allowed investment by thermal player. However, in some jurisdictions market capacity could be expanded by constructing new dams. Also, in some jurisdictions investment in other technologies, like green technologies, have occurred. For example, capacity investments in wind farms in Germany and Denmark are becoming an important source of power generation. An interesting extension of our model would be to incorporate investment in all types of power generation technologies, which we leave for future research.

\section{References}

[1] S.Ambec, J. Doucet. Decentralizing Hydro Power Production. Canadian Journal of Economics, 36(3):587-607, 2003.

[2] J. Bushnell. A mixed complementarity model of hydrothermal electricity competition in the western United States. Operations Research, 51(1):80-93, 2003.

[3] J. Bushnell and J. Ishii. Equilibrium model of investment in restructured electricity markets. mimeo, December 2006.

[4] C. Crampes and M. Moreaux. Water resource and power generation. International Journal of Industrial Organization, 19(6):975-997, May 2001.

[5] P. Cramton and S. Stoft.The Convergence of Market Designs for Adequate Generating Capacity. Working Paper, 72 pages, April 2006. http://www.ksg.harvard.edu/hepg/Papers/Cramton_Stoft_0406.pdf

[6] Garcia, A., J. Reitzes, E. Stacchetti. Strategic Pricing When Electricity is Storable. Journal of Regulatory Economics, 20(3), 223-247, 2001.

[7] A. Garcia and J. Shen. Equilibrium capacity expansion under stochastic demand growth. Universtity of Virgina, 2007.

[8] A. Garcia and E. Stacchetti. Investment dynamics in electricity markets. mimeo, 2008.

[9] T. Genc, S.S. Reynolds, and S. Sen. Dynamic oligopolistic games under uncertainty: A stochastic programming approach. Journal of Economic Dynamics and Control, $31: 55-80,2007$. 
[10] T. Genc and S. Sen. An analysis of capacity and price trajectories for the Ontario electricity market using dynamic Nash equilibrium under uncertainty. Energy Economics, 30(1):173-191, 2008.

[11] A. Haurie, G. Zaccour, and Y. Smeers. Stochastic equilibrium programming for dynamic oligopolistic markets. Journal of Optimization Theory and Applications, 66, 1990.

[12] A. Haurie and F. Moresino. S-adapted oligopoly equilibria and approximations in stochastic variational inequalities. Annals of Operations Research, 114:183-2001, 2002.

[13] F. H. Murphy and Y. Smeers. Generation capacity expansion in imperfectly competitive restructured electricity markets. Operations Research, 53(4):646-661, 2005.

[14] P. Joskow. Competitive Electricity Markets and Investment in New Generating Capacity. The New Energy Paradigm (Dieter Helm, Editor), Oxford University Press, 2007.

[15] P.O. Pineau and P. Murto. An oligopolistic investment model of the Finnish electricity market. Annals of Operations Research, 121, 2003.

[16] F. Roques, D. Newbery and W. Nuttall (2005). Investment incentives and electricity market design: the British experience. Review of Network Economics, 4(3), 2005.

[17] T.J. Scott and E.G. Read. Modeling hydro reservoir operation in a deregulated electricity market. International Transactions in Operational Research, 3(3/4):243-53, 1996. 